\title{
Age, diabetes mellitus, and dialysis modality are associated with risk of poor muscle strength and physical function in hemodialysis and peritoneal dialysis patients
}

\author{
Maryanne Zilli Canedo Silva ${ }^{1}$, Karina Jesus Antonio ${ }^{1}$, João Marcos Soares Reis ${ }^{2}$, Leticia Salmazzo Alves ${ }^{2}$, \\ Jacqueline Costa Teixeira Caramori ${ }^{1}$, Barbara Perez Vogt ${ }^{3}$
}

${ }^{1}$ Department of Internal Medicine, Botucatu Medical School, São Paulo State University (UNESP), Botucatu, Brazil

${ }^{2}$ Nutrition Course, Health Sciences School, University of Western São Paulo (UNOESTE), Presidente Prudente, Brazil

${ }^{3}$ Faculty of Medicine, Graduate Program in Health Sciences, Federal University of Uberlândia (UFU), Uberlândia, Brazil

Background: Due to the poor outcomes associated with the impairment of physical function and muscle strength in patients on maintenance dialysis, it is important to understand the factors that may influence physical function and muscle strength. The aim of this study was to explore the factors associated with physical function in hemodialysis and peritoneal dialysis patients.

Methods: Patients with chronic kidney disease on dialysis for at least 3 months, aged 18 years old or above, were enrolled. Physical function was assessed by handgrip strength, gait and sit-to-stand tests, and the Short Physical Performance Battery (SPPB). Clinical and laboratory data were collected to verify the association with physical function parameters through binary logistic regression.

Results: One-hundred ninety patients on maintenance dialysis were included; 140 patients (73.7\%) on hemodialysis and 50 (26.3\%) on peritoneal dialysis. The mean age was $57.3 \pm 14.9$ years, 109 (57.4\%) were male, and 87 (45.8\%) were older than 60 years. The median SPPB was 8.0 points (6.0-10.0 points) and the mean \pm standard deviation of handgrip strength was $24.7 \pm 12.2 \mathrm{~kg}$. Binary logistic regression showed that age, type of renal replacement therapy, diabetes mellitus, and serum creatinine were significantly associated with both higher 4-meter gait test times and lower SPPB scores. Only age and diabetes mellitus were associated with higher sit-to-stand test times, while age and ferritin were associated with lower handgrip strength.

Conclusion: Age, diabetes mellitus, serum creatinine, and hemodialysis modality are factors related to physical function in dialysis patients.

Keywords: Chronic renal insufficiency, Dialysis, Hand strength, Physical functional performance, Physical performance

Received: August 25, 2020; Revised: January 5, 2021; Accepted: January 18, 2021

Editor: Chan-Duck Kim, Kyungpook National University, Daegu, Republic of Korea

Correspondence: Barbara Perez Vogt

Medical School (FAMED), Federal University of Uberlândia (UFU), Campus Umuarama, Av. Pará, 1720, Bloco 2U, Uberlândia, Minas Gerais 38405320, Brazil. E-mail: barbaravogt@ufu.br

ORCID: https://orcid.org/0000-0002-2619-0354

Copyright (C) 2021 by The Korean Society of Nephrology

(a) This is an Open Access article distributed under the terms of the Creative Commons Attribution Non-Commercial and No Derivatives License (http:// creativecommons.org/licenses/by-nc-nd/4.0/) which permits unrestricted non-commercial use, distribution of the material without any modifications, and reproduction in any medium, provided the original works properly cited. 


\section{Introduction}

The prevalence of sarcopenia in chronic kidney disease (CKD) patients varies widely, ranging from $4.0 \%$ to $13.7 \%$ depending on the CKD stage, kidney replacement therapy (KRT), and the consensus version [1-3]. This number is even higher in CKD patients aged 60 years and above [3].

In this context, the assessment of physical function of CKD patients undergoing conservative treatment or who are on dialysis is extremely important. Deterioration of physical function is accelerated in patients with CKD, increasing the risk of worse outcomes, such as loss of independence, risk of morbidity, reduced quality of life, and reduced survival $[4,5]$. Recently, the association of poor physical function with mortality was verified in meta-analyses including nondialysis [5] and dialysis CKD patients, with an inverse association found between handgrip strength (HGS) and all-cause mortality [6].

Due to the importance of physical function and muscle strength for patients on maintenance dialysis, it is important to understand the factors that may influence these traits. As a consequence, patients at risk of diminished physical function and muscle strength may receive more attention from their health care team in order to improve or maintain such attributes. Therefore, our objective was to explore the factors associated with muscle strength and physical function in maintenance hemodialysis (HD) and peritoneal dialysis (PD) patients.

\section{Methods}

This was a cross-sectional analysis, which included participants with CKD on HD or PD from three protocols. These patients were either on (1) maintenance HD or PD in the Dialysis Unit from the Clinics Hospital of Botucatu Medical School (Botucatu, Brazil) or (2) maintenance HD in the Dialysis Service from the Presidente Prudente Regional Hospital (Presidente Prudente, Brazil). The three research protocols were approved by the respective Institution Research Ethics Committees (CAAE 71393717.7.0000.5411, 61634816.4.0000.5411, and 73640317.9.0000.5515) and the enrolled patients provided written informed consent.

The enrolled patients were on dialysis for at least 3 months and aged 18 years old or above. Patients who were bedridden, those with upper and lower limb sequelae, or amputees were excluded because they cannot perform the necessary physical performance assessments. Patients with other catabolic conditions were also excluded, such as neoplasia, final stage liver disease, severe heart diseases, or chronic obstructive pulmonary disease.

Age, sex, dialysis vintage, presence of diabetes mellitus $(\mathrm{DM})$, dialysis adequacy $(\mathrm{Kt} / \mathrm{V})$, and routine laboratory tests (serum levels of urea, creatinine, albumin, cholesterol, triglycerides, hemoglobin, calcium, phosphorus, potassium, parathyroid hormone, iron, and ferritin) were collected from medical records.

Anthropometric evaluation consisted of actual body weight and height measurements for body mass index calculations. For this assessment, patients on PD had an empty peritoneal cavity and HD patients were evaluated after their dialysis session.

Muscle strength and physical function were evaluated in this study using a 4-meter gait test, sit-to-stand test, Short Physical Performance Battery (SPPB), and HGS test. All assessments were performed 30 minutes after the end of the HD session. Patients on PD were evaluated during routine care visits.

To assess the 4-meter gait test, patients had two attempts to walk a 4-meter course at their usual speed with static start. Each attempt was timed and the faster value was considered for the analyses [7]. The sit-to-stand test measures the amount of time the patient takes to rise five times from a straight-backed chair. Patients were instructed to stand up from the initial sitting position and sit down five times as quickly as possible, without using his or her arms [7]. Sit-tostand tests require both muscle strength and endurance [8].

The SPPB consists of three different tests of lower-extremity function; balance test (ability to maintain for 10 seconds the following stand positions: feet together side-by-side, semi-tandem, and tandem), 4-meter gait test, and sit-tostand chair test. A summary score ranging from 0 (worst performance) to 12 (best performance) was calculated [7].

HGS measurement was performed using a Jamar (Sammons Preston Rolyan, Bolingbrook, IL, USA) or Saehan hydraulic dynamometer (Saehan Corp., Changwon, Korea). These dynamometers are considered equivalents, with an intraclass correlation coefficient of $>0.97$ [9]. Patients were positioned with the dynamometer facing away from the body and the other arm extended to the side of the body. In the sequence, patients were instructed to hold the grip 
for around 3 seconds with maximum force in response to a voice command. Three measurements were performed, with intervals of around 30 seconds between each; the highest value was considered for analysis. The evaluation of patients on HD was performed on the side opposite from their arteriovenous fistula or central access. Patients on PD were evaluated through the dominant limb [10].

\section{Statistical analysis}

Data are expressed as mean \pm standard deviation or median (interquartile range), according to the variables' distribution. Frequencies are expressed as percentages. In order to compare patients according to age, presence of DM, and method of dialysis, Student t test, Mann-Whitney test, or chi-square test were used.

The correlations between clinical and laboratory variables with muscle strength and physical function were assessed by Spearman or Pearson correlation coefficients. To address associated factors, four models of binary logistic regression were built, each one with a muscle strength or physical function parameter as a dependent variable (4-meter gait time, sit-to-stand test time, SPPB total score, and HGS) categorized by the respective median. HGS was categorized according to the median by each sex. Parameters correlated with the dependent variables $(\mathrm{p}<0.2)$ in univariate analysis were selected to be included in the logistic regression models.

Statistical significance was considered when $\mathrm{p}<0.05$. Analyses were performed using IBM SPSS version 22.0 (IBM Corp., Armonk, NY, USA).

\section{Results}

One-hundred ninety patients on maintenance dialysis were enrolled, with 140 patients (73.7\%) on HD and $50(26.3 \%)$ on PD. The PD modality used by most patients was continuous cycling PD (CCPD) $(60.0 \%, \mathrm{n}=30)$, followed by Nocturnal Intermittent PD modality ( $36.0 \%, \mathrm{n}=18)$, and continuous ambulatory PD modality $(4.0 \%, \mathrm{n}=2)$. The majority of the patients $(57.4 \%)$ were males, and 87 individuals (45.8\%) were older than 60 years. Demographic, clinical, and laboratory characteristics, as well as physical function scores of the patients, are presented in Table 1.

Patients were compared according to dialysis method. Dialysis vintage, serum urea, creatinine, albumin, and potassi-
Table 1. Characteristics of the enrolled patients

\begin{tabular}{|c|c|}
\hline Characteristic & Data \\
\hline Patients & 190 \\
\hline Age (yr) & $57.3 \pm 14.9$ \\
\hline \multicolumn{2}{|l|}{ Sex } \\
\hline Female & $81(42.6)$ \\
\hline Male & $109(57.4)$ \\
\hline Diabetes mellitus & $60(31.6)$ \\
\hline Dialysis vintage (mo) & $20.6(8.8-59.7)$ \\
\hline \multicolumn{2}{|l|}{$\mathrm{Kt} / \mathrm{V}^{\mathrm{a}}$, /wk } \\
\hline PD & $2.2 \pm 0.5$ \\
\hline HD & $1.3 \pm 0.3$ \\
\hline Weight (kg) & $70.3 \pm 15.4$ \\
\hline Body mass index $\left(\mathrm{kg} / \mathrm{m}^{2}\right)$ & $26.4 \pm 4.9$ \\
\hline \multicolumn{2}{|l|}{ SPPB score } \\
\hline Balance test & $4.0(3.0-4.0)$ \\
\hline 4-Meter gait test & $3.0(2.0-4.0)$ \\
\hline Sit-to-stand test & $1.0(1.0-2.0)$ \\
\hline SPPB total score & $8.0(6.0-10.0)$ \\
\hline 4-Meter gait test $(\mathrm{sec})^{b}$ & $5.2(4.2-6.6)$ \\
\hline Sit-to-stand test $(\mathrm{sec})^{c}$ & $17.6(14.3-21.7)$ \\
\hline Handgrip strength (kg) & $24.7 \pm 12.2$ \\
\hline Male & $31.1 \pm 11.5$ \\
\hline Female & $16.0 \pm 6.4$ \\
\hline Urea (mg/dL) & $120.1 \pm 36.4$ \\
\hline HD & $127.4 \pm 37.2$ \\
\hline PD & $99.5 \pm 24.7$ \\
\hline Creatinine (mg/dL) & $10.0 \pm 3.2$ \\
\hline HD & $10.3 \pm 3.2$ \\
\hline PD & $9.0 \pm 2.8$ \\
\hline Albumin (g/dL) & $3.8 \pm 0.4$ \\
\hline Hemoglobin (g/dL) & $11.1 \pm 1.7$ \\
\hline Calcium (mg/dL) & $9.1 \pm 0.8$ \\
\hline Phosphorus (mg/dL) & $5.4 \pm 1.4$ \\
\hline Potassium (mmol/L) $)^{d}$ & $5.1 \pm 0.9$ \\
\hline Iron ( $\mu \mathrm{g} / \mathrm{dL})$ & $64.0(49.8-91.0)$ \\
\hline Ferritin $(\mu \mathrm{g} / \mathrm{dL})$ & $278.7(133.2-573.3)$ \\
\hline PTH (pg/mL) & $297.8(175.1-592.5)$ \\
\hline \multicolumn{2}{|l|}{ Cholesterol (mg/dL) } \\
\hline Total & $143.4 \pm 38.1$ \\
\hline HDL & $37.0(31.0-47.0)$ \\
\hline $\mathrm{LDL}^{\mathrm{e}}$ & $71.0 \pm 31.0$ \\
\hline Triglycerides (mg/dL) & $143.0(98.0-205.5)$ \\
\hline
\end{tabular}

Data are expressed as number only, mean \pm standard deviation, number (\%), or median (interquartile range). HD, hemodialysis; HDL, high-density lipoprotein; LDL, low-density lipoprotein; PD, peritoneal dialysis; PTH, parathyroid hormone; SPPB, Short Physical Performance Battery.

${ }^{a} n=182(P D, 48 ; H D, 134),{ }^{d} n=184,{ }^{e} n=187 .{ }^{b}$ Four patients were not able to perform the 4-meter gait test. 'Thirty-two patients were not able to perform the sit-to-stand test. 
um were significantly higher in HD patients. Serum calcium, iron, ferritin, and high-density lipoprotein (HDL)-cholesterol were significantly lower in HD patients.

Comparison of physical function variables between patients according to age (younger or older than 60 years), presence of $\mathrm{DM}$, and dialysis method (PD and HD) are presented in Table 2.

There was a significant correlation among all physical function parameters; the time of the 4-meter gait test was positively correlated with the sit-to-stand test $(\mathrm{r}=0.17$, $\mathrm{p}$ $=0.02$ ) and negatively correlated both with the SPPB total score $(\mathrm{r}=-0.72, \mathrm{p}<0.001)$ and HGS $(\mathrm{r}=-0.41, \mathrm{p}<0.001)$. The sit-to-stand test was negatively correlated with SPPB total score $(\mathrm{r}=-0.15, \mathrm{p}=0.04)$. The SPPB total score was positively correlated with HGS $(\mathrm{r}=0.47, \mathrm{p}<0.001)$. Correlations between physical function parameters and other variables are presented in Table 3.

For the binary logistic regression, times for the 4-meter gait test were categorized by the median, 5.22 seconds. Sex, age, type of KRT, dialysis vintage, DM, serum creatinine, urea, phosphate, and ferritin were included in the model. Sit-to-stand test times were categorized by the median of 17.56 seconds and SPPB was categorized by its median of 8 points. The same variables were included in each model; sex, age, type of KRT, DM, serum creatinine, urea, ferritin, albumin, and HDL-cholesterol.

HGS was categorized according to the median for each sex; $30 \mathrm{~kg}$ for male and $15 \mathrm{~kg}$ for female. The variables included in the model were sex, age, dialysis vintage, DM, Kt/ $\mathrm{V}$, serum creatinine, urea, calcium, potassium, hemoglobin, ferritin, albumin, and HDL-cholesterol.

The variables included in the final model were those that improved the model predictive capacity. In the final models, age, type of KRT (HD), DM, and serum creatinine were significantly associated with both a higher 4-meter gait test time and lower SPPB (models 1 and 3, respectively) (Table 4). Only age and DM were associated with a higher sit-to-stand test time (model 2), while age and ferritin were associated with a lower HGS (model 4) (Table 4).

\section{DISCUSSION}

The objective of this study was to identify the factors that are associated with muscle strength and physical function, assessed by four parameters in patients receiving dialysis treat- ment for at least 3 months. The results indicate that aging, DM, lower serum creatinine, and HD modality are factors related to poor muscle strength and physical function. Age was associated with all muscle strength and physical function tests performed in the study. DM, HD modality, and serum creatinine were associated with three of the four tests.

Aging is a well-known feature associated with worsening muscle strength and physical function in the general population [11]. Moreover, a natural decrease in muscle mass, strength, and performance occurs with aging; however, multiple other conditions contribute to such decrease [12]. Aging is considered a condition that leads to a proinflammatory state. The increased cytokine levels affect muscle protein synthesis, leading to muscle mass loss [13]. Lower muscle strength has been associated with increased interleukin- 6 and C-reactive protein levels in older adults [14]. CKD uremia and dialysis are also known to promote inflammation, which may act synergistically with the effects of 'inflammaging' and lead to poor physical function.

In fact, among dialysis patients, older patients present worse physical function compared to younger patients $[15,16]$. Our results have shown age as an independent predictor of all parameters used to assess muscle strength and physical function in this study. Moreover, the decline in physical function related to aging often leads to loss of independence and ability to perform activities of daily life, as well as poorer quality of life [17]. Poor physical function is associated with increased risk of outcomes such as cognitive impairment, institutionalization, falls [18], disability [7], cardiovascular events [19], and mortality [7,20]. Therefore, elderly people on KRT should receive greater attention from their health care team and interventions should be proposed.

One of the comorbidities affecting physical function is DM. In our results, DM was associated with SPPB and the sit-to-stand and gait tests, but not with HGS. DM may affect muscle function through several mechanisms. Peripheral insulin resistance decreases the glucose uptake to muscle and reduces muscle tissue anabolic rates [13]. Also, changes in microvascularization decrease blood flow to the muscles. Moreover, other prevalent comorbidities (such as decreased vision, heart failure, neuropathy, and peripheral vascular disease) result in decreased physical activity and may also explain decreased physical function among the dialysis population $[21,22]$. However, the prevalence of these elements was not assessed in the current study. 


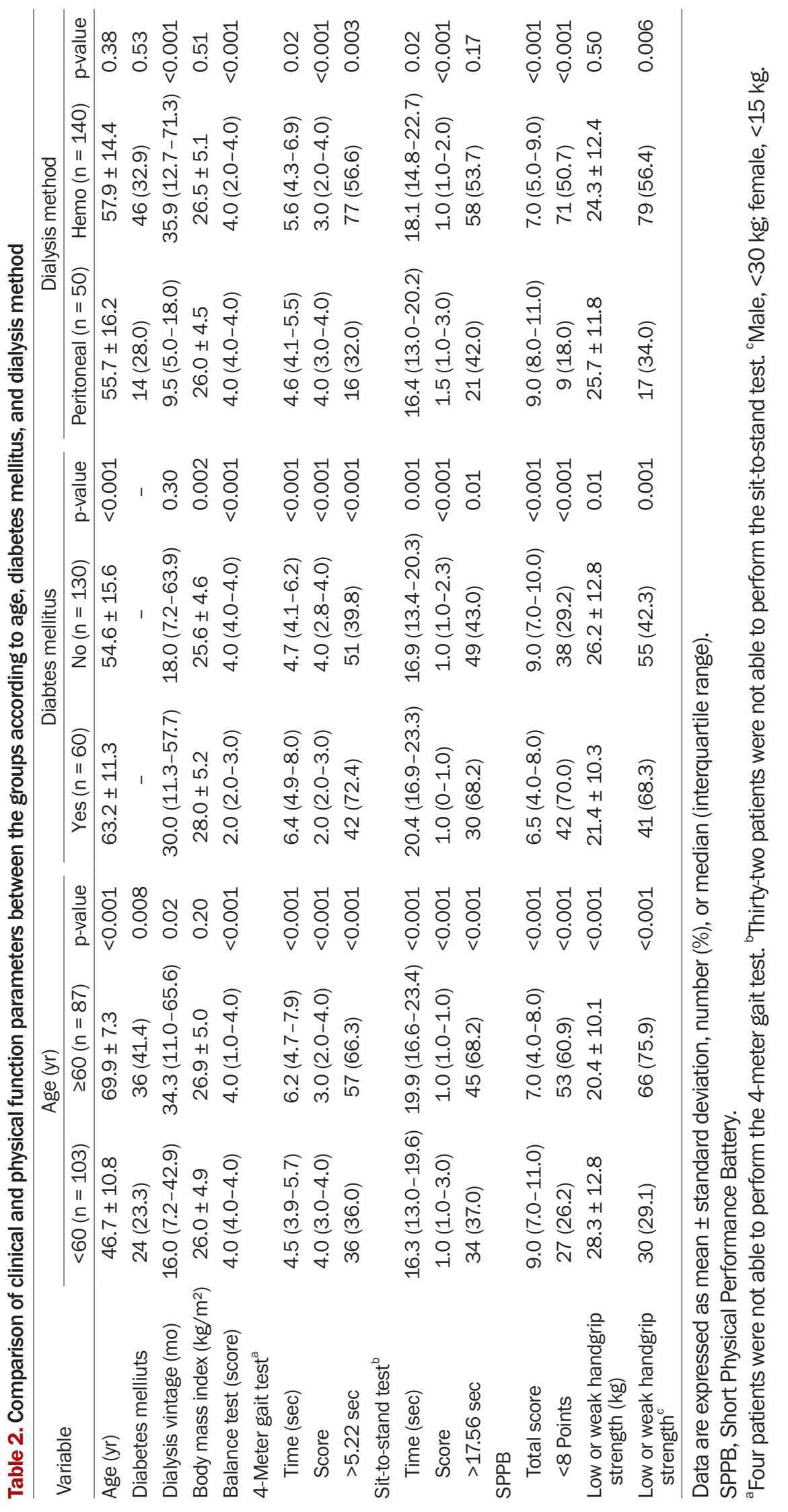


Table 3. Correlation of physical function with different variables

\begin{tabular}{|c|c|c|c|c|c|c|c|c|}
\hline \multirow{2}{*}{ Variable } & \multicolumn{2}{|c|}{ 4-Meter gait test (sec) } & \multicolumn{2}{|c|}{ Sit-to-stand (sec) } & \multicolumn{2}{|c|}{ SPPB (total score) } & \multicolumn{2}{|c|}{ Handgrip strength (kg) } \\
\hline & $r$ & $\mathrm{p}$ & $r$ & $p$ & $r$ & $\mathrm{p}$ & $r$ & $\mathrm{p}$ \\
\hline Age & 0.49 & $<0.001$ & 0.45 & $<0.001$ & -0.51 & $<0.001$ & -0.38 & $<0.001$ \\
\hline Dialysis vintage & 0.22 & 0.002 & -0.05 & 0.50 & -0.30 & $<0.001$ & -0.18 & 0.01 \\
\hline $\mathrm{Kt} / \mathrm{V}$ & 0.05 & 0.52 & -0.02 & 0.79 & 0.07 & 0.36 & -0.19 & 0.01 \\
\hline Creatinine & -0.31 & $<0.001$ & -0.20 & 0.01 & 0.32 & $<0.001$ & 0.40 & $<0.001$ \\
\hline Calcium & 0.07 & 0.35 & 0.07 & 0.36 & -0.03 & 0.68 & -0.15 & 0.04 \\
\hline Phosphorus & -0.17 & 0.02 & -0.09 & 0.25 & 0.13 & 0.08 & -0.02 & 0.82 \\
\hline Parathyroid hormone & -0.09 & 0.23 & -0.04 & 0.62 & 0.03 & 0.64 & 0.04 & 0.63 \\
\hline Potassium & 0.02 & 0.79 & 0.03 & 0.76 & -0.05 & 0.50 & 0.20 & 0.007 \\
\hline Iron & -0.08 & 0.29 & -0.04 & 0.66 & 0.18 & 0.01 & 0.10 & 0.16 \\
\hline $\mathrm{HDL}$ & -0.03 & 0.67 & -0.14 & 0.07 & 0.07 & 0.34 & -0.13 & 0.08 \\
\hline LDL & 0.01 & 0.87 & 0.03 & 0.70 & 0.03 & 0.71 & -0.11 & 0.15 \\
\hline Triglycerides & -0.02 & 0.77 & -0.04 & 0.60 & 0.05 & 0.54 & 0.10 & 0.19 \\
\hline Hemoglobin & 0.09 & 0.24 & 0.02 & 0.82 & -0.08 & 0.33 & 0.11 & 0.14 \\
\hline Albumin & 0.00 & 0.98 & 0.14 & 0.08 & -0.08 & 0.28 & 0.17 & 0.02 \\
\hline
\end{tabular}

HDL, high-density lipoprotein; LDL, low-density lipoprotein; SPPB, Short Physical Performance Battery.

Table 4. Binary logistic regressions with physical function parameters as dependent variables

\begin{tabular}{|c|c|c|c|c|}
\hline Model & Dependent variable & Independent variable & OR $(95 \% \mathrm{Cl})$ & p-value \\
\hline \multirow[t]{4}{*}{1} & 4-Meter gait test & Age & $1.07(1.04-1.11)$ & $<0.001$ \\
\hline & $>5.22 \sec (n=186)$ & Dialysis modality (HD) & $5.13(2.09-12.62)$ & $<0.001$ \\
\hline & & Diabetes mellitus & $2.63(1.19-5.79)$ & 0.02 \\
\hline & & Serum creatinine & $0.85(0.74-0.97)$ & 0.02 \\
\hline \multirow[t]{3}{*}{2} & Sit-to-stand test & Age & $1.07(1.03-1.10)$ & $<0.001$ \\
\hline & $>17.56 \mathrm{sec}(\mathrm{n}=157)$ & Diabetes mellitus & $2.31(1.04-5.11)$ & 0.04 \\
\hline & & Male & $1.77(0.85-3.69)$ & 0.13 \\
\hline \multirow[t]{4}{*}{3} & SPPB & Age & $1.12(1.07-1.17)$ & $<0.001$ \\
\hline & $<8$ points $(n=186)$ & Dialysis modality (HD) & $20.13(5.69-71.18)$ & $<0.001$ \\
\hline & & Diabetes mellitus & $4.73(1.90-11.78)$ & 0.001 \\
\hline & & Serum creatinine & $0.74(0.61-0.88)$ & 0.001 \\
\hline \multirow[t]{2}{*}{4} & Handgrip strength & Age & $1.14(1.10-1.19)$ & $<0.001$ \\
\hline & $<30 \mathrm{~kg}$ in male or $<15 \mathrm{~kg}$ in female & Ferritin & $1.001(1.00-1.003)$ & 0.004 \\
\hline
\end{tabular}

$\mathrm{Cl}$, confidence interval; HD, hemodialysis; SPPB, Short Physical Performance Battery; OR, odds ratio.

Serum creatinine was a significant predictor of physical function in the models with the 4-meter gait test and SPPB as dependent variables. Serum creatinine may reflect muscle mass of patients on dialysis [23]. Muscle function declines at a rate different from that of muscle size [24], and the worse physical function of patients on HD compared to non-CKD elderly was not explained by muscle size [22]. Nonetheless, a recent systematic review discusses how the association between physical function and muscle size in CKD patients is still controversial [25].

The current study found that HD patients have a higher risk of reduced muscle strength and poor physical function 
compared to PD patients. In a systematic review, Purnell et al. [26] showed no significant differences in physical function in $76 \%$ of comparisons between these two KRT modalities. However, the majority of the included studies used 36-Item Short Form Survey (SF-36) domains to assess physical function; such methodology is considered a subjective assessment. A comparison of quality of life between $\mathrm{HD}$ and PD patients using SF-36 has not found differences between the KRT modalities [27]. Moreover, none of the studies included in the systematic review [26] used the same objective assessments used in the current study (gait test, sit-to-stand test, SPPB, or HGS).

The characteristics of each KRT modality may influence physical function through the daily routine imposed by the treatment. Most PD patients have some free time during the day, with one or two dialysate changes per day on CCPD modality. On the other hand, HD patients spend many hours, 3 days a week, with no activities during transit to the HD center and during the HD session. Moreover, frequent symptoms after HD sessions, such as bleeding, hypotension, dizziness, fatigue, etc., increase the need for rest. Thus, these factors related to HD therapy may favor a more sedentary lifestyle, and intradialytic interventions aimed at physical function improvement could be useful.

Another issue related to KRT modality is the assessment timing. In our study, HD patients were assessed after the $\mathrm{HD}$ session. At this time, hypotension, dizziness, and fatigue may affect the results of the physical function tests. However, before the dialysis session, the patients are overhydrated, which may also influence the results of the performance tests [28]. Pinto et al. [29] compared HGS before and after HD sessions and concluded that the HD procedure negatively affects HGS. Moreover, HGS variation was correlated with blood pressure variations. On the other hand, Leal et al. [30] found no difference between HGS values before and after HD sessions. Dialysis variables, such as ultrafiltration, inter-dialytic body weight gain, $\mathrm{Kt} / \mathrm{V}$, urea, and blood pressure were not correlated with HGS values [30]. Some studies assessed physical function before [31,32] or after [10,33] dialysis session, and others on nondialysis days [22]. Therefore, there is no standard for physical function assessment timing.

Assessments of muscle strength and physical function can be done by different methods in CKD. Sit-to-stand tests, 4-meter gait speed tests, SPPB, and HGS were chosen for this study based on the ease and practicality of performing these tests, even in dialysis unit facilities. These tests reflect common daily living activities, i.e., getting up from a chair or walking small distances. SPPB and HGS assessments were previously shown to be reliable in HD patients $[31,32]$ and both have been recommended by the European Working Group on Sarcopenia in Older People 2 (EWGSOP2) for sarcopenia diagnosis [8]. However, there are no specific cutoffs for physical function assessments among the CKD population.

In a comparison between patients with low and appropriate muscle strength, Isoyama et al. [34] observed that those with low strength were older and had more comorbidities, such as cardiovascular disease and DM, lower serum creatinine, and higher inflammatory markers levels. Furthermore, HGS has been considered an independent predictor of allcause mortality in HD and PD patients [10,34], which was recently confirmed by Hwang et al. [6] in a meta-analysis. They found that patients with low HGS had a 1.88-fold higher risk of death than those with higher HGS. In addition, a 1-kg unit increase in HGS was associated with a 5\% reduction in the risk of mortality [6].

As poor physical function is associated with poorer outcomes in the dialysis population, interventions that improve physical function may decrease the risk of poor outcomes. Exercise training is the most investigated intervention, and several modalities have been tested.

A recent meta-analysis [35] reported that either aerobic or resistance exercise modalities could improve objective measures of physical function in patients undergoing dialysis. Additionally, intradialytic exercise improved physical function more efficiently than interdialytic exercise, supporting the hypothesis of intradialytic exercise interventions for HD patients [35]. Intradialytic resistance band exercise training and neuromuscular electrical stimulation were also effective interventions to enhance physical function in patients on HD [35-37]. It is important to highlight that previous studies have shown exercise interventions are safe and well-tolerated [36], with no significant changes in hemodynamic parameters [38] and no adverse events during the training session [39].

There are fewer studies with exercise interventions in PD. Although the physical function of these patients is better than in HD patients, exercise could bring other benefits to this population as well as to HD patients. In a randomized controlled trial in PD patients, a 12-week home-based 
program that included aerobic and resistance exercise was effective in improving aerobic capacity, some domains of quality of life, serum albumin, and insulin resistance [40].

Combined interventions, such as nutritional supplementation and exercise, offer strategies to improve muscle strength and physical function. Martin-Alemañy et al. [41] showed that the combination of oral nutritional supplementation with aerobic or resistance intradialytic exercise had better effects on physical function than supplementation alone in young HD patients. Due to the association of vitamin D status with muscle health and the high prevalence of vitamin D deficiency in CKD, Olvera-Soto et al. [42] added cholecalciferol supplements to a resistance exercise program intervention in stage 4 CKD patients. A significant increase in HGS was observed. Those strategies are still incipient in CKD research. Therefore, more trials are necessary to assess such effects.

A limitation of this study is the merging of three different data sets of HD and PD patients from two centers. Nonetheless, in all of these data sets, the assessments were obtained using the same protocol. The cross-sectional design may also be a limitation since it is not possible to demonstrate the relationship between cause and effect.

In conclusion, age, DM, and dialysis modality are factors related to muscle strength and physical function in dialysis patients. Thus, special attention should be given to patients with these characteristics, and specific interventions should be tested with the objective to improve muscle strength and physical function in both HD and PD patients.

\section{Conflicts of interest}

All authors have no conflicts of interest to declare.

\section{Funding}

This work has been supported by São Paulo Research Foundation (FAPESP) grant (No. 2017/13187-2).

\section{Acknowledgments}

We acknowledge all patients from the Dialysis Unit of the Clinics Hospital of Botucatu Medical School and Dialysis Section of the Regional Hospital of Presidente Prudente who took part in the study. We also thank Luciana K. Camargos
Batista, Camila Z. G. Freitas, Simone C. C. Buchalla, and Vanessa Mota Silva for their contributions in data collection.

\section{Authors' contributions}

Conceptualization: MZCS, KJA, JCTC, BPV

Data curation, Investigation: MZCS, KJA, JMSR, LSA

Formal analysis: MZCS, BPV

Funding acquisition: JMSR, BPV

Project administration: JCTC, BPV

Writing-original draft: MZCS, BPV

Writing-review \& editing: KJA, JMSR, LSA, JCTC

All authors read and approved the final manuscript.

\section{ORCID}

Maryanne Zilli Canedo Silva, https://orcid.org/0000-0001-66023625

Karina Jesus Antonio, https://orcid.org/0000-0002-7806-1407

João Marcos Soares Reis, https://orcid.org/0000-0002-0311$309 \mathrm{X}$

Leticia Salmazzo Alves, https://orcid.org/0000-0002-8065-8544 Jacqueline Costa Teixeira Caramori, https://orcid.org/00000002-0093-9515

Barbara Perez Vogt, https://orcid.org/0000-0002-2619-0354

\section{References}

1. da Silva MZ, Vogt BP, Reis NS, Caramori JCT. Update of the European consensus on sarcopenia: what has changed in diagnosis and prevalence in peritoneal dialysis? Eur J Clin Nutr 2019;73:1209-1211.

2. Souza VA, Oliveira D, Barbosa SR, et al. Sarcopenia in patients with chronic kidney disease not yet on dialysis: Analysis of the prevalence and associated factors. PLoS One 2017;12:e0176230.

3. Ren H, Gong D, Jia F, Xu B, Liu Z. Sarcopenia in patients undergoing maintenance hemodialysis: incidence rate, risk factors and its effect on survival risk. Ren Fail 2016;38:364-371.

4. Koufaki P, Kouidi E. Current best evidence recommendations on measurement and interpretation of physical function in patients with chronic kidney disease. Sports Med 2010;40:1055-1074.

5. MacKinnon HJ, Wilkinson TJ, Clarke AL, et al. The association of physical function and physical activity with all-cause mortality and adverse clinical outcomes in nondialysis chronic kidney disease: a systematic review. Ther Adv Chronic Dis 2018;9:209- 
226.

6. Hwang SH, Lee DH, Min J, Jeon JY. Handgrip strength as a predictor of all-cause mortality in patients with chronic kidney disease undergoing dialysis: a meta-analysis of prospective cohort studies. J Ren Nutr 2019;29:471-479.

7. Guralnik JM, Simonsick EM, Ferrucci L, et al. A short physical performance battery assessing lower extremity function: association with self-reported disability and prediction of mortality and nursing home admission. J Gerontol 1994;49:M85-M94.

8. Cruz-Jentoft AJ, Bahat G, Bauer J, et al. Sarcopenia: revised European consensus on definition and diagnosis. Age Ageing 2019;48:16-31.

9. Reis MM, Arantes PM. Assessment of hand grip strength- validity and reliability of the Saehan dynamometer. Fisioter Pesqui 2011;18:176-181.

10. Vogt BP, Borges MC, Goés CR, Caramori JC. Handgrip strength is an independent predictor of all-cause mortality in maintenance dialysis patients. Clin Nutr 2016;35:1429-1433.

11. Guralnik JM, Fried LP, Salive ME. Disability as a public health outcome in the aging population. Annu Rev Public Health 1996;17:25-46.

12. Brioche T, Pagano AF, Py G, Chopard A. Muscle wasting and aging: experimental models, fatty infiltrations, and prevention. Mol Aspects Med 2016;50:56-87.

13. Evans WJ, Paolisso G, Abbatecola AM, et al. Frailty and muscle metabolism dysregulation in the elderly. Biogerontology 2010; 11:527-536.

14. Cesari M, Penninx BW, Pahor M, et al. Inflammatory markers and physical performance in older persons: the InCHIANTI study. J Gerontol A Biol Sci Med Sci 2004;59:242-248.

15. Uchiyama K, Washida N, Muraoka K, et al. Exercise capacity and association with quality of life in peritoneal dialysis patients. Perit Dial Int 2019;39:66-73.

16. Greco A, Paroni G, Seripa D, Addante F, Dagostino MP, Aucella F. Frailty, disability and physical exercise in the aging process and in chronic kidney disease. Kidney Blood Press Res 2014;39:164168.

17. Fragala MS, Kenny AM, Kuchel GA. Muscle quality in aging: a multi-dimensional approach to muscle functioning with applications for treatment. Sports Med 2015;45:641-658.

18. Abellan van Kan G, Rolland Y, Andrieu S, et al. Gait speed at usual pace as a predictor of adverse outcomes in community-dwelling older people an International Academy on Nutrition and Aging (IANA) Task Force. J Nutr Health Aging 2009;13:881-889.

19. Bellettiere J, Lamonte MJ, Unkart J, et al. Short physical performance battery and incident cardiovascular events among older women. $J$ Am Heart Assoc 2020;9:e016845.

20. Pavasini R, Guralnik J, Brown JC, et al. Short Physical Performance Battery and all-cause mortality: systematic review and meta-analysis. BMC Med 2016;14:215.

21. McKee A, Morley JE, Matsumoto AM, Vinik A. Sarcopenia: an endocrine disorder? Endocr Pract 2017;23:1140-1149.

22. Marcus RL, LaStayo PC, Ikizler TA, et al. Low physical function in maintenance hemodialysis patients is independent of muscle mass and comorbidity. J Ren Nutr 2015;25:371-375.

23. Kalantar-Zadeh K, Streja E, Molnar MZ, et al. Mortality prediction by surrogates of body composition: an examination of the obesity paradox in hemodialysis patients using composite ranking score analysis. Am J Epidemiol 2012;175:793- 803.

24. Carrero JJ, Johansen KL, Lindholm B, Stenvinkel P, Cuppari L, Avesani CM. Screening for muscle wasting and dysfunction in patients with chronic kidney disease. Kidney Int 2016;90:53-66.

25. Tarca BD, Wycherley TP, Bennett P, Meade A, Ferrar KE. Modifiable physical factors associated with physical functioning for patients receiving dialysis: a systematic review. J Phys Act Health 2020;17:475-489.

26. Purnell TS, Auguste P, Crews DC, et al. Comparison of life participation activities among adults treated by hemodialysis, peritoneal dialysis, and kidney transplantation: a systematic review. Am J Kidney Dis 2013;62:953-973.

27. Ramos EC, Santos Ida S, Zanini Rde V, Ramos JM. Quality of life of chronic renal patients in peritoneal dialysis and hemodialysis. J Bras Nefrol 2015;37:297-305.

28. Carlos C, Grimes B, Segal M, Johansen K. Predialysis fluid overload and gait speed: a repeated measures analysis among patients on chronic dialysis. Nephrol Dial Transplant 2020;35:1027-1031.

29. Pinto AP, Ramos CI, Meireles MS, Kamimura MA, Cuppari L. Impact of hemodialysis session on handgrip strength. J Bras Nefrol 2015;37:451-457.

30. Leal VO, Stockler-Pinto MB, Farage NE, et al. Handgrip strength and its dialysis determinants in hemodialysis patients. Nutrition 2011;27:1125-1129.

31. Segura-Ortí E, Martínez-Olmos FJ. Test-retest reliability and minimal detectable change scores for sit-to-stand-to-sit tests, the six-minute walk test, the one-leg heel-rise test, and handgrip strength in people undergoing hemodialysis. Phys Ther 2011; 91:1244-1252.

32. Ortega-Pérez de Villar L, Martínez-Olmos FJ, Junqué-Jiménez A, et al. Test-retest reliability and minimal detectable change scores for the short physical performance battery, one-legged 
standing test and timed up and go test in patients undergoing hemodialysis. PLoS One 2018;13:e0201035.

33. Carrero JJ, Chmielewski M, Axelsson J, et al. Muscle atrophy, inflammation and clinical outcome in incident and prevalent dialysis patients. Clin Nutr 2008;27:557-564.

34. Isoyama N, Qureshi AR, Avesani CM, et al. Comparative associations of muscle mass and muscle strength with mortality in dialysis patients. Clin J Am Soc Nephrol 2014;9:1720-1728.

35. Clarkson MJ, Bennett PN, Fraser SF, Warmington SA. Exercise interventions for improving objective physical function in patients with end-stage kidney disease on dialysis: a systematic review and meta-analysis. Am J Physiol Renal Physiol 2019;316:F856-F872.

36. Bullani R, El-Housseini Y, Giordano F, et al. Effect of intradialytic resistance band exercise on physical function in patients on maintenance hemodialysis: a pilot study. J Ren Nutr 2011;21:6165.

37. Valenzuela PL, Morales JS, Ruilope LM, de la Villa P, Santos-Lozano A, Lucia A. Intradialytic neuromuscular electrical stimulation improves functional capacity and muscle strength in people receiving haemodialysis: a systematic review. J Physio- ther 2020;66:89-96.

38. Corrêa H, Neves R, Silva J, et al. Exercise prior to hemodialysis session does not induce hemodynamic instability in patients on chronic hemodialysis. J Exerc Physiol Online 2019;22:109-117.

39. Castro AP, Barbosa SR, Mansur HN, Ezequiel DG, Costa MB, Paula RB. Intradialytic resistance training: an effective and easy-to-execute strategy. J Bras Nefrol 2018;41:215-223.

40. Uchiyama K, Washida N, Morimoto K, et al. Home-based aerobic exercise and resistance training in peritoneal dialysis patients: a randomized controlled trial. Sci Rep 2019;9:2632.

41. Martin-Alemañy G, Espinosa-Cuevas ML, Pérez-Navarro M, et al. Effect of oral nutritional supplementation with and without exercise on nutritional status and physical function of adult hemodialysis patients: a parallel controlled clinical trial (AVANTE-HEMO Study). J Ren Nutr 2020;30:126-136.

42. Olvera-Soto MG, Ruiz Medina VL, Medeiros M, et al. Effect of resistance exercise plus cholecalciferol on nutritional status indicators in adults with stage 4 chronic kidney disease. J Ren Nutr 2020;30:232-241. 